\title{
Predictors of osteoarthritis following operative treatment of medial tibial plateau fractures
}

\section{Parkkinen, Markus}

2018-02

Parkkinen , M , Lindahl , J , Mäkinen , T J , Koskinen , S K, Mustonen , A \& Madanat , R 2018 , ' Predictors of osteoarthritis following operative treatment of medial tibial plateau fractures ' , Injury , vol. 49 , no. 2 , pp. 370-375 . https://doi.org/10.1016/j.injury.2017.11.014

http://hdl.handle.net/10138/300575

https://doi.org/10.1016/j.injury.2017.11.014

publishedVersion

Downloaded from Helda, University of Helsinki institutional repository.

This is an electronic reprint of the original article.

This reprint may differ from the original in pagination and typographic detail.

Please cite the original version. 
Full length article

\title{
Predictors of osteoarthritis following operative treatment of medial tibial plateau fractures ${ }^{\text {ts }}$
}

\author{
Markus Parkkinen $^{\mathrm{a}, *}$, Jan Lindahl ${ }^{\mathrm{a}}$, Tatu J. Mäkinen ${ }^{\mathrm{a}}$, Seppo K. Koskinen ${ }^{\mathrm{c}}$, \\ Antti Mustonen $^{\mathrm{b}}$, Rami Madanat ${ }^{\mathrm{a}}$ \\ a Department of Orthopaedics and Traumatology, Helsinki University Hospital, Topeliuksenkatu 5, 00260 Helsinki, Finland \\ ${ }^{\mathrm{b}}$ Pohjola Sairaala, Department of Radiology, Puutarhurinkuja 2, 00300 Helsinki, Finland \\ ${ }^{\mathrm{c}}$ Trauma and Musculoskeletal Imaging, Karolinska University Hospital, Stockholm, Sweden
}

\section{A R T I C L E I N F O}

Keywords:

Medial plateau

Tibia fracture

Tibial plateau

Schatzker IV

Osteoarthritis

\begin{abstract}
A B S T R A C T
Purpose: To determine factors influencing the development of posttraumatic osteoarthritis (OA) following medial tibial plateau fractures and to evaluate concomitant injuries associated with these fractures.

Materials and methods: A chart review of patients with operatively treated medial tibial plateau fractures admitted to our Level I trauma centre from 2002 to 2008 was performed. Of 63 patients, 41 participated in a clinical and radiographic examination. The mean age was 47 years (range 16-78) and the mean followup time was 7.6 (range 4.7-11.7) years. All patients had preoperative computed tomography (CT) scans and postoperative radiographs. At the end of follow-up, standing radiographs, mechanical axis, and CT scans were evaluated.

Results: Of the 41 patients, 24 had no or mild (Kellgren-Lawrence grade 0-2) OA and 17 had severe (grade 3-4) OA. Initial articular depression measured from preoperative CT scans was a significant predictor of OA (median $1.8 \mathrm{~mm}$ vs $4.5 \mathrm{~mm}, \mathrm{p}=0.009$ ). Fracture line extension to the lateral plateau $(\mathrm{p}=0.68)$ or fracture comminution $(\mathrm{p}=0.21)$ had no effect on the development of posttraumatic OA, nor did articular depression at the end of follow-up $(\mathrm{p}=0.68)$ measured from CT scans. Mechanical axis $>4^{\circ}$ of varus and $\geq 2 \mathrm{~mm}$ articular depression or step-off were associated with worse WOMAC pain scores, but did not affect other functional outcome scores. Six patients (10\%) had permanent peroneal nerve dysfunction. Ten patients (16\%) required LCL reconstruction and nine (14\%) ACL avulsions were treated at the time of fracture stabilisation.

Conclusions: The amount of articular depression measured from preoperative CT scans seems to predict the development of posttraumatic OA, probably reflecting the severity of chondral injury at the time of fracture. Restoration of mechanical axis and articular congruence are important in achieving a good clinical outcome.
\end{abstract}

(c) 2017 Elsevier Ltd. All rights reserved.

\section{Introduction}

Fractures of the medial tibial plateau are rare. Even though these fractures comprise only $10-20 \%$ of all proximal tibial fractures, they generally have the worst prognosis [1]. These fractures are usually a consequence of varus stress of the knee along with axial loading [2]. The medial plateau carries about $60 \%$

\footnotetext{
ts Investigation performed at the Department of Orthopaedics and Traumatology, University of Helsinki and Helsinki University Hospital, Helsinki, Finland.

* Corresponding author.

E-mail address: markus.parkkinen@hus.fi (M. Parkkinen).
}

of the bodyweight and consequently has, as compared to the lateral plateau, a denser subchondral bone [3]. This structural difference, combined with the anatomical valgus axis of the knee and natural tendency of external impact laterally, makes the lateral side more prone to fractures. Medial plateau fractures thus usually are thought to result from a high-energy trauma, unlike more common lateral plateau fractures [1,4]. However, in one recent study half of the medial plateau fractures were caused by lowenergy trauma [5].

Medial plateau fractures have a high prevalence of concomitant injuries. Of these, lateral collateral ligament (LCL) and anterior cruciate ligament $(\mathrm{ACL})$ injuries are the most common, as are peroneal nerve injuries [6,7]. Many authors recommend operative 
treatment of medial plateau fractures if there is any displacement in the fracture $[4,8-10]$. The premise is that since the medial plateau carries a higher proportion of the knee's load than the lateral side, it might cause these fractures to dislocate more easily. Especially, posterior shearing fractures of the plateau are prone to dislocate. There is still little data on the medium and long-term outcomes following operative treatment of these fractures. Some evidence indicates that the quality of the medial plateau fracture reduction, measured from plain $\mathrm{x}$-rays, affects the resulting functional outcome [9].

The primary objective of this study was to determine fractureand surgery-related factors that could influence the development of posttraumatic osteoarthritis (OA). Our hypothesis was that residual articular incongruence is related to the development of $\mathrm{OA}$. The secondary objective was to evaluate functional outcome and the type of concomitant injuries associated with these fractures. The local Ethical Committee approved the study and we obtained informed consent from each patient.

\section{Materials and methods}

We performed a chart review of all patients with operatively treated tibial plateau fractures who were admitted to our Level I trauma centre between 1 January 2002 and 31 December 2008. A total of 389 patients with proximal tibia fractures were identified in the hospital database. We reviewed knee radiographs and routine multi-detector computed tomography (CT) scans with axial, sagittal, and coronal reformations and classified fractures according to the AO/OTA [11] classification system. From this cohort, we identified 63 patients with AO/OTA type 41-B1.2, B1.3, B3.2, and B3.3 medial plateau fractures (Fig. 1). All but one patient had preoperative CT scans for accurate evaluation of fracture morphology. Fourteen of these 63 patients also had a preoperative MRI examination. The indication for operative treatment at our institution was any displacement of the medial tibial plateau fracture. We reviewed hospital records, including operative reports and clinical notes, and determined the nature of the injuries and complications of either the fracture or its treatment.

The most common mechanisms of injury were same-level falls in 28 patients and road traffic accidents in 14 patients. Other frequent injury mechanisms included the following: bicycle accident in 9 patients, sports-related injury in 8 patients, automobile collision with a pedestrian in 2 patients, and falls from a height greater than $1 \mathrm{~m}$ in 2 patients.

\section{Operative technique}

All patients were treated using the standard techniques of exposure, open reduction, and stable internal fixation. Twenty-two patients had a temporary external fixation until swelling was reduced and the remainder had a splint while awaiting surgery. The most common approach for fracture reduction was posteromedial (52 patients, 83\%) and 13 of these patients also had an additional anterolateral approach performed [12]. Eight patients were operated from an anterior approach, two patients were operated from an anterolateral approach only, and one was treated using a percutaneous technique. Ten patients had an additional lateral approach used for lateral collateral ligament (LCL) reconstruction. Thirty-four fractures were stabilized using angular stable plates and 22 using traditional buttress plates. Seven patients had only screw fixation. The postoperative management protocol included early mobilization using a hinged knee brace. All patients were instructed to be non-weight bearing for the first six to ten weeks, followed by partial weight bearing for a further two to six weeks depending on the operating surgeon's intraoperative assessment of fracture comminution and stability.

\section{Clinical and functional evaluation}

Of the 63 patients, 41 were able to participate in a follow-up visit. The patient characteristics are summarized in Table 1. Two patients underwent total knee arthroplasty and thus were not included in the clinical and functional evaluations. Seven patients completed functional evaluation information forms, but were unable to attend the follow-up visit. Fifteen patients were excluded from the study due to no follow-up data (Fig. 2). The mean followup time was 7.6 (range 4.7-11.7) years.

One orthopaedic surgeon (MP) examined patients at the followup visit. Valgus and varus laxity were evaluated using manual testing in extension and in $30^{\circ}$ of flexion. To assess anterior laxity, the Lachman, anterior drawer, and pivot shift tests were used. Posterior laxity was evaluated using the posterior drawer test. Range of motion was measured using a goniometer. Results were compared to the uninjured contralateral knee. Patients completed two validated functional outcome measurement tools; the Modified Lysholm knee score and the Western Ontario and McMaster Universities Osteoarthritis index (WOMAC) [13,14].

\section{Radiological analysis}

For the current study, weight-bearing knee radiographs were obtained at the follow-up visit to evaluate the stage of posttraumatic OA according to the Kellgren-Lawrence classification (grade 0 to 4 ) [15]. Radiographs were obtained with the knees in (neutral) extension and the tibias in neutral rotation. Full-lenght radiographs of the lower extremities were taken to evaluate the mechanical axis. Computed tomography (CT) (GE Discovery CT750 HD, General Electric Medical Systems, Milwaukee, WI, USA) of the injured knee was obtained at the end of follow-up to measure the depression of the joint surface. Axial 1.25-mm thick reconstructions and 2-mm sagittal and coronal reformation were used for the analysis. The residual depression of the joint surface was also measured from the first postoperative conventional non-weight bearing radiographs. The images were independently evaluated on clinical PACS workstations (IMPAX DS 3000, version 4.5, Agfa-

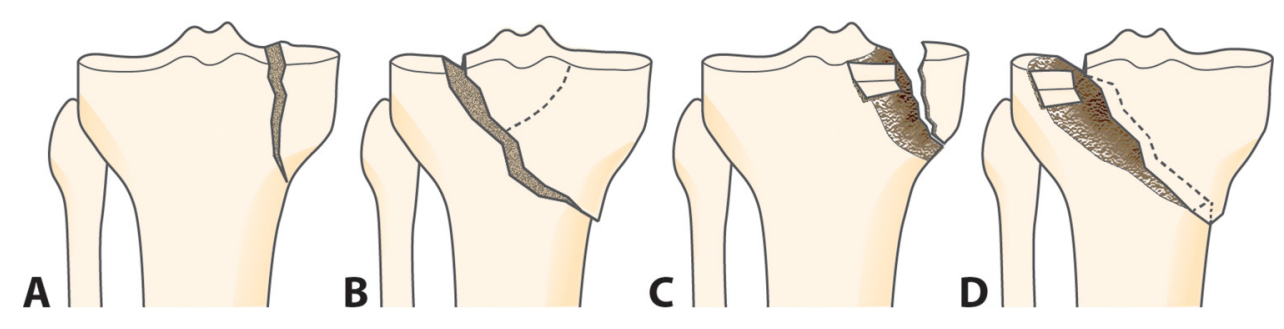

Fig. 1. Medial tibial plateau fracture subtypes $(A=B 1.2, B=B 1.3, C=B 3.2$, and $D=B 3.3$ ) according to $A O / O T A$ classification. 
Table 1

Characteristics of 41 patients with medial condyle fractures.

\begin{tabular}{|c|c|c|c|}
\hline Characteristics & Patients (\%) with no or mild OA $(\mathrm{n}=24)^{\mathrm{a}}$ & Patients (\%) with moderate of severe OA $(n=17)^{b}$ & $\mathrm{p}$ value \\
\hline Age, mean (range), years & $46(16-78)$ & $47(29-73)$ & 0.89 \\
\hline Mean body mass index (range), $\mathrm{kg} / \mathrm{m}^{2}$ & $27(20-38)$ & $29(21-59)$ & 0.59 \\
\hline Male gender, n (\%) & $18(75)$ & $8(47)$ & 0.067 \\
\hline ASA $^{\mathrm{c}}$ class 3 or $4, \mathrm{n}(\%)$ & $4(17)$ & $3(18)$ & 1.00 \\
\hline Fracture type, n (\%) & & & 0.41 \\
\hline B1.2 & $2(8)$ & 0 & \\
\hline B1.3 & $4(17)$ & $1(6)$ & \\
\hline B3.2 & $9(37.5)$ & $6(35)$ & \\
\hline B3.3 & $9(37.5)$ & $10(59)$ & \\
\hline Open fracture, n (\%) & $1(4)$ & $1(6)$ & 1.00 \\
\hline
\end{tabular}

a Kellgren-Lawrence grade $0-2$.

b Kellgren-Lawrence grade 3-4.

c American Society of Anesthesiologists.

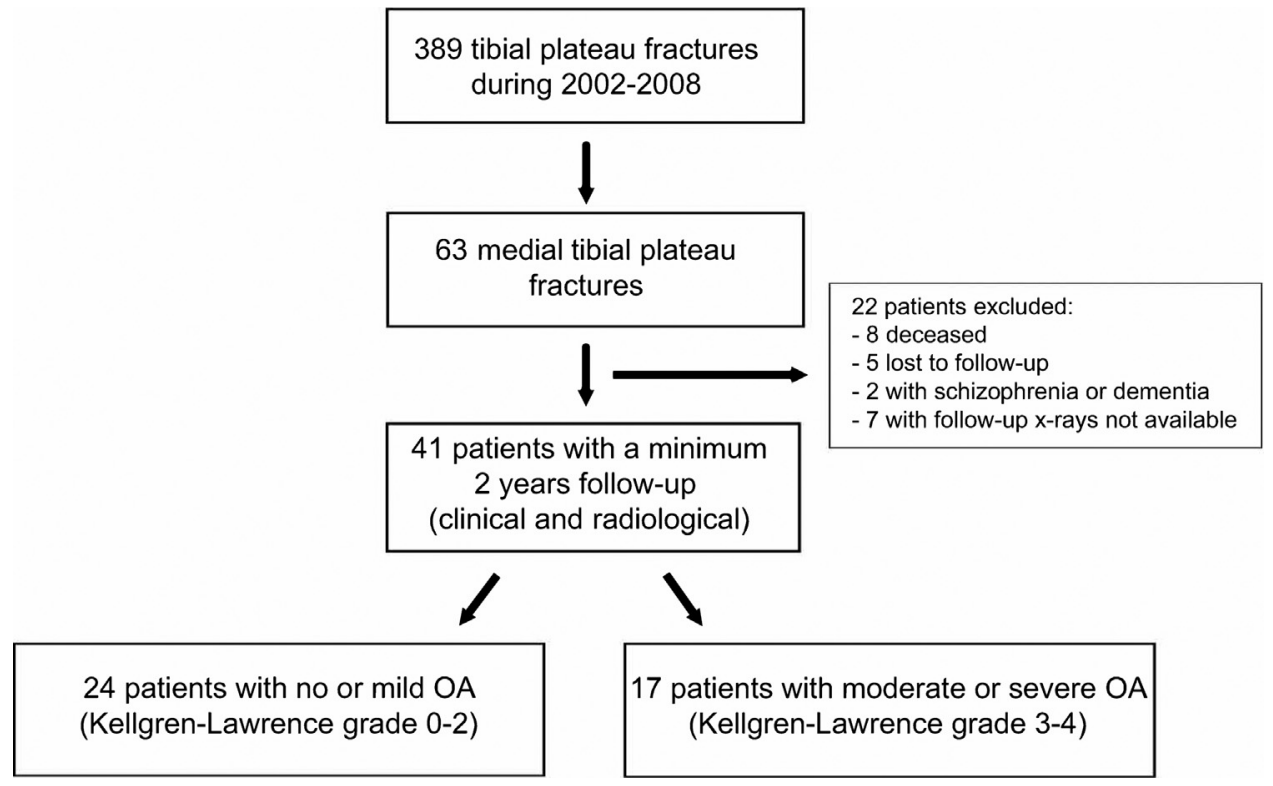

Fig. 2. A flow chart of the inclusion of patients with operatively treated tibia medial plateau fractures.

Table 2

Radiological results of 41 patients with medial condyle fractures.

\begin{tabular}{|c|c|c|c|}
\hline & 24 patients with no or mild $\mathrm{OA}^{\mathrm{a}}$ & 17 patients with moderate of severe $\mathrm{OA}^{\mathrm{b}}$ & p-value \\
\hline Involvement of lateral condyle, n (\%) (B1.3, B3.3) & $14(58)$ & $11(65)$ & 0.68 \\
\hline Comminuted fracture (B3.2, B3.3) & $18(75)$ & $16(94)$ & 0.21 \\
\hline Median articular depression in preoperative CT scan (range), mm & $1.8(0-8.5)$ & $4.5(0-9.4)$ & 0.0093 \\
\hline Median articular widening in preoperative CT scan (range), $\mathrm{mm}$ & $2.5(0-15.5)$ & $2.5(0-17)$ & 0.75 \\
\hline Median articular depression in first postoperative x-ray (range), $\mathrm{mm}$ & $0.0(0-4.2)$ & $1.0(0-3.9)$ & 0.18 \\
\hline Median articular depression at the end of follow-up in CT scan (range), $\mathrm{mm}$ & $0.8(0-3.4)$ & $1.2(0-7)^{\mathrm{c}}$ & 0.68 \\
\hline Median mechanical axis (range) & 1.3 varus ( 8.1 valgus-11.8 varus) & 5.0 varus $^{\mathrm{c}}$ (4.8 valgus-9.7 varus) & 0.36 \\
\hline
\end{tabular}

a Kellgren-Lawrence grade $0-2$

b Kellgren-Lawrence grade 3-4.

c Data for 38 patients ( 2 patients with total knee arthroplasty and 1 patient with no postoperative CT or mechanical axis images excluded). 
Gevaert N.V., Mortsel, Belgium) by two musculoskeletal radiologists, and mean measurement values were used.

\section{Statistical analysis}

An independent biostatistician performed the statistical analysis of the data. The differences in categorical variables between patients with or without posttraumatic OA were analysed with chi-square test or Fisher's exact test. The mean ages between patient groups were compared using two-sample $t$-test. The Mann-Whitney $U$ test was used to test the difference in nonnormally distributed continuous variables between patients. Receiver-operating characteristics (ROC) analysis and Youden's (sensitivity + specificity-1) index were used to find the optimal cutoff value for depression measured from preoperative CT scans in predicting posttraumatic OA. The maximum value of the Youden's index was used as a criterion for selecting the optimal cut-off value. Statistical analyses were performed with SAS System for Windows, version 9.4 (SAS Institute INC, Cary, NC, USA). P values $<0.05$ were considered statistically significant.

\section{Results}

\section{Radiological results}

Of the 41 patients, 24 had no or mild (Kellgren-Lawrence grade $0-2$ ) OA and 17 had severe (grade 3-4) OA. Radiological results of 41 patients with follow-up x-rays are shown in Table 2. Initial articular depression measured from preoperative CT scans was a significant predictor of posttraumatic OA ( $p=0.009)$ (Figs. 3 and 4$)$. The optimal cut-off value for depression was $\geq 3.4 \mathrm{~mm}$ in predicting posttraumatic OA (sensitivity 0.81 , specificity 0.75 , positive predictive value 0.68 , and negative predictive value 0.86 ) (Fig. 5). Area under the ROC curve was 0.74 .

Patients with median mechanical axis $>4^{\circ}$ of varus had significantly more WOMAC pain $(\mathrm{p}=0.045)$, but there was no difference in WOMAC stiffness $(p=0.85)$, WOMAC function $(\mathrm{p}=0.13)$, and Modified Lysholm $(\mathrm{p}=0.16)$ scores compared to patients with $\leq 4^{\circ}$ of varus. The difference in mechanical axis compared to the uninjured leg was not associated with an increased risk of posttraumatic OA $(p=0.10)$. Patients with articular depression or step-off $\geq 2 \mathrm{~mm}$ in the first postoperative radiographs had more WOMAC pain $(\mathrm{p}=0.044)$, but no difference in WOMAC stiffness $(p=0.83)$, WOMAC function $(p=0.12)$, and Modified Lysholm $(\mathrm{p}=0.96)$ scores.

\section{Additional injuries and complications}

Six out of 63 patients had permanent peroneal nerve dysfunction. There were no vascular injuries. Six patients developed compartment syndrome necessitating fasciotomy. Ten patients (16\%) had LCL repair or reconstruction performed at the time of fracture stabilisation; 7 out of 20 (35\%) with isolated medial plateau fractures (B1.2, B3.2) and 3 out of 43 (7\%) with an oblique fracture type (B1.3, B3.3). The diagnosis of LCL injury was based on clinical evaluation after stabilization of the medial plateau fracture. Seven patients also had MRI. Patients who had LCL reconstruction or repair performed had similar risk for OA as patients without $\mathrm{LCL}$ injury $(p=1.00)$. Nine patients $(14 \%)$ also had a displaced ACL avulsion fixation performed. One patient had delayed ACL reconstruction and one patient had delayed $\mathrm{ACL}$ and $\mathrm{PCL}$ reconstruction due to subjective instability and cruciate ligament injury seen on MRI.

There were two open fractures. One was Gustilo grade I and the other was grade IIIB, which required gastrocnemius muscle flap coverage at the time of fracture stabilisation. The patient with the grade IIIB open fracture developed a persistent infection and the osteosynthesis material was removed after fracture healing one year from the index operation. This patient did not develop posttraumatic OA at 6 years follow-up time.

\section{Functional results after follow-up}

The functional results of 39 patients with follow-up visits and the seven patients without follow-up x-rays are presented in Table 3. At the follow-up visit, three patients demonstrated a minor extension deficit $\left(0-5^{\circ}\right)$. Fifteen patients had minor flexion deficit $\left(5-15^{\circ}\right)$, and eight patients had flexion deficit of $20-30^{\circ}$. All the patients who had ACL avulsion fixed at the time of fracture stabilization had stable ACL at the clinical examination. At the follow-up, two patients had residual ACL laxity, one patient PCL laxity and one patient both ACL and PCL laxity with no cruciate ligament reconstruction. These patients had no subjective instability, thus no surgical treatment was warranted. One patient, who had delayed ACL and PCL reconstruction done, also had minor residual PCL laxity. Three patients had mild grade I MCL laxity, and none had LCL instability. The mean Lysholm score of the six patients with peroneal dysfunction was 72 (range 51-87), the mean WOMAC pain score was 16 (range 2-57), stiffness score was 22 (range 8-66) and function score 19 (range 0-55).
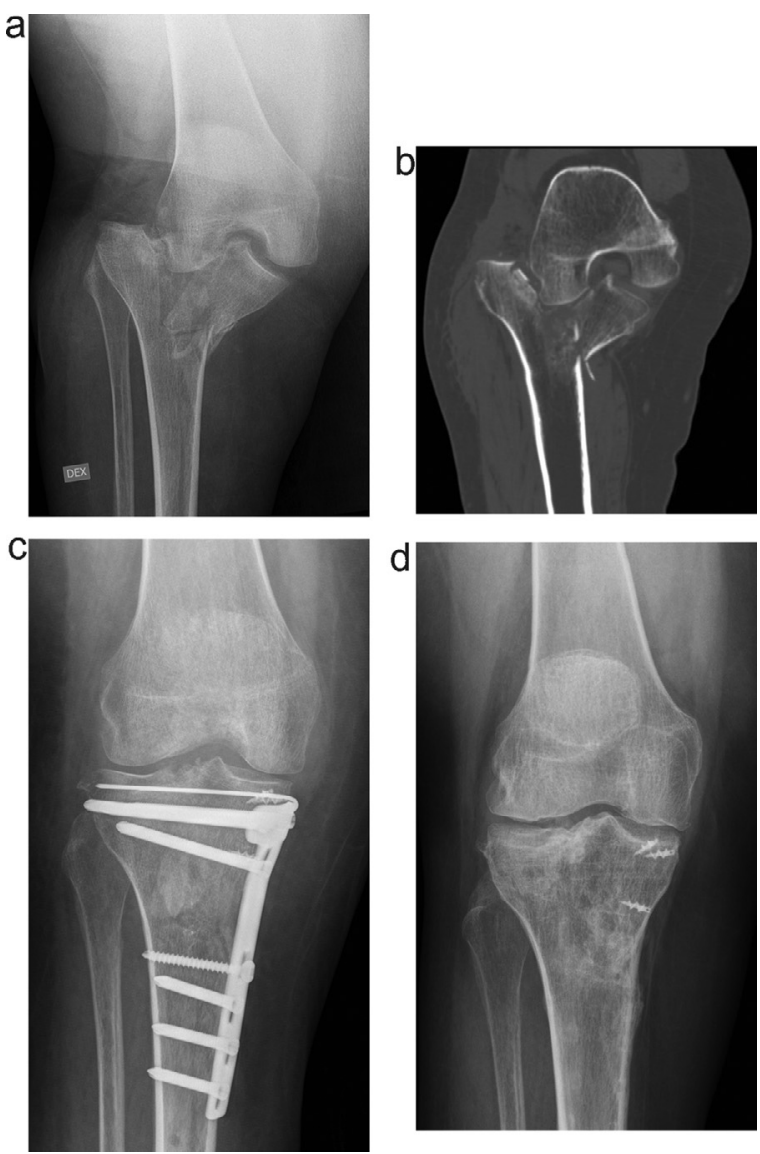

Fig. 3. Radiographs of a 41-year old woman who sustained a medial tibial plateau fracture after falling from a bicycle (a). Pre-operative CT (coronal view) showed marked articular depression (b). ORIF with medial locking plate was performed (c). Follow-up standing radiograph shows severe post-traumatic OA (K-G grade 3) (d). 

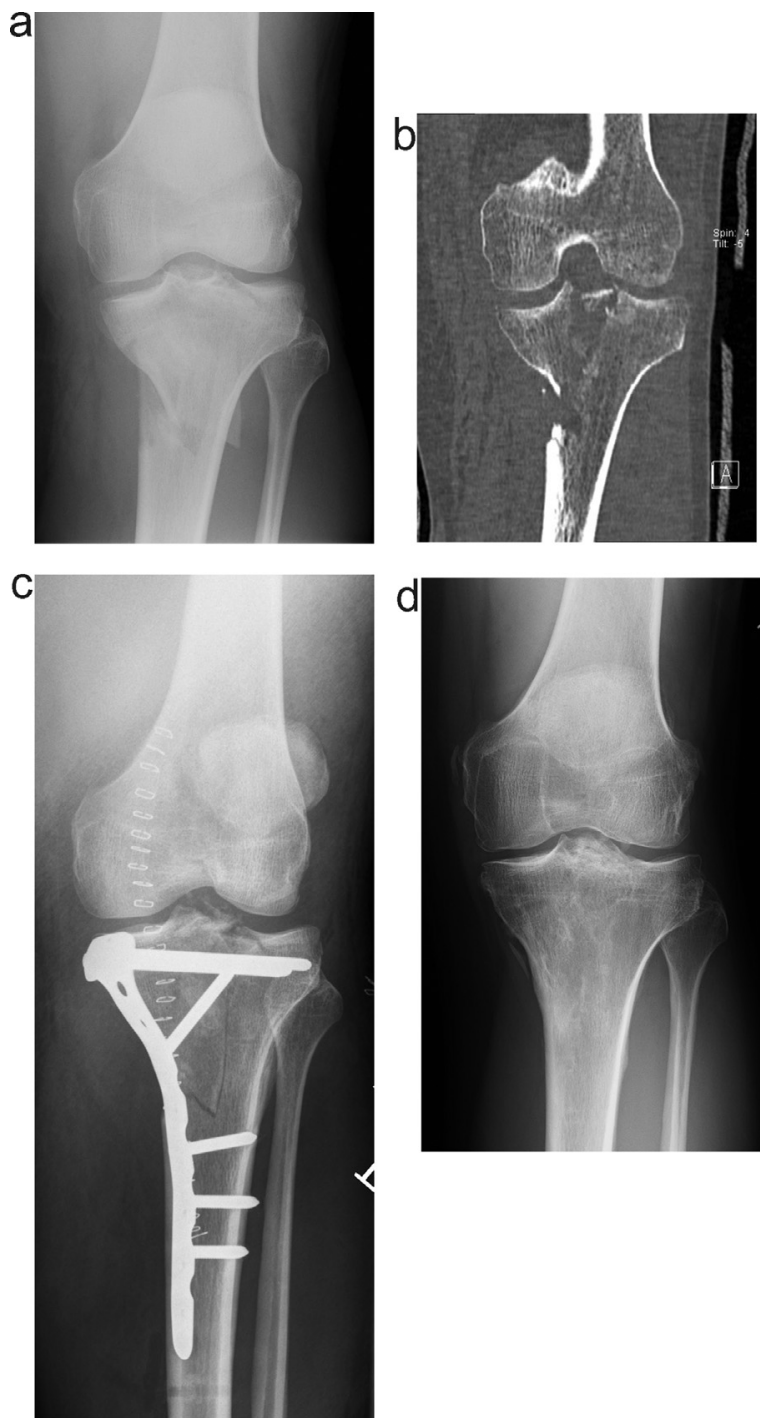

Fig. 4. Radiographs of a 33-year old male who sustained a medial tibial plateau fracture resulting from a motorcycle accident (a). Pre-operative CT (coronal view) showed minimal articular depression at the tibia (b). ORIF with medial locking plate was performed (c). Follow-up standing radiograph shows minimal post-traumatic OA (K-G grade 1$)(d)$.

\section{Discussion}

The primary objective of this study was to determine fractureand surgery-related factors that could influence the development of posttraumatic osteoarthritis (OA). Our hypothesis

was that residual articular incongruence is related to the development of OA. According to previous studies, about $20-44 \%$ of the patients will develop posttraumatic OA after a proximal tibia

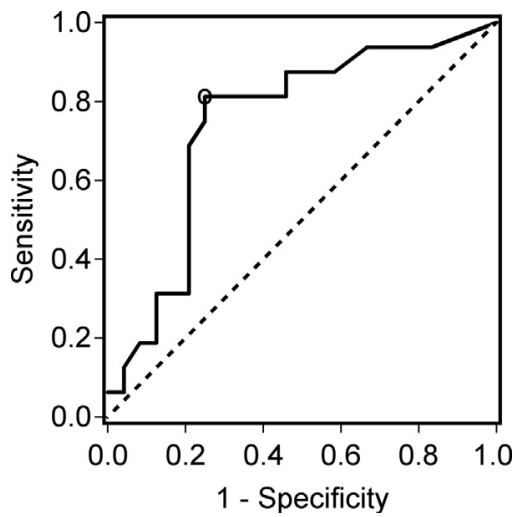

Fig. 5. Receiver operator curve for depression measured from preoperative computed tomography (CT) scans to predict the development of posttraumatic OA (area under the curve $=0.74$ ). The optimal cut-off value (circle in figure) for depression measured from preoperative CT scans was $\geq 3.4 \mathrm{~mm}$ (sensitivity 0.81 , specificity 0.75 , positive predictive value 0.68 and negative predictive value 0.86 ).

fracture [16-19]. Yet, only 7\% of the patients developed posttraumatic arthritis needing arthroplasty after 10 years of follow-up after the injury [20]. In our study, 17 out of 41 patients (41\%) developed Kellgren-Lawrence grade $3-4$ posttraumatic OA after a mean 7.6 years of follow-up. Two of these patients underwent total knee arthroplasty because of severely painful arthritis. Development of posttraumatic OA may be caused by initial trauma to the tibial plateau cartilage [21-23] or may develop due to an altered axial loading pattern to the tibial plateau caused by articular incongruence or malalignment $[8,24,25]$. In the current study, the initial depression of the fracture as seen on the preoperative CT scan predicted the development of OA. The fracture subtype according to AO/OTA classification did not seem to predict the early progress of OA. The quality of postoperative reduction, measured from the first postoperative $\mathrm{x}$-ray and CT scan at the end of followup, did not predict the development of posttraumatic OA between the two groups.

The second objective was to evaluate functional outcomes and the type of concomitant injuries associated with these fractures. The functional outcome after open reduction and internal fixation of medial plateau fractures were generally good. The median WOMAC scores were comparable to population-based normative values [26]. The functional outcome results were worse in the group with Kellgren-Lawrence grade 3-4 OA.

Concomitant injuries related to medial plateau fractures are common. Gardner et al. reported in their magnetic resonance imaging (MRI) analyses that the incidence of complete LCL rupture was $57 \%$ in Schatzker IV fractures $(n=7)$ [7]. It should be noted that MRI might over-diagnose clinically relevant soft tissue injuries in the presence of fracture [27]. Kannus et al. have reported unsatisfactory results of grade III LCL ruptures treated conservatively [28]. Primary repair of LCL is indicated for bony avulsions; however, midsubstance tears should be treated with ligament

Table 3

Functional results of patients with medial condyle fractures.

\begin{tabular}{|c|c|c|c|c|}
\hline & 24 patients with no or mild $\mathrm{OA}$ & 15 patients with moderate or severe $\mathrm{OA}^{\mathrm{a}}$ & 7 patients with no follow-up x-rays & p value ${ }^{b}$ \\
\hline \multicolumn{5}{|l|}{ Median WOMAC (range) } \\
\hline Pain & $5(0-57)$ & $7(0-60)$ & $2(0-11)$ & 0.34 \\
\hline Stiffness & $4(0-66)$ & $12(0-77)$ & $2(0-9)$ & 0.10 \\
\hline Function & $3(0-55)$ & $18(0-63)$ & $2(0-5)$ & 0.02 \\
\hline Median Lysholm (range) & $89(51-100)$ & $69(35-99)$ & $87(67-100)$ & 0.04 \\
\hline
\end{tabular}

a 2 patients who underwent total knee arthroplasty excluded.

b Mann-Whitney $U$ test; patients with moderate or severe OA compared with no or mild OA. 
reconstruction [29]. In our series, 10 patients (16\%) had grade III LCL rupture at the clinical evaluation necessitating reconstruction. None of the patients at the follow-up visit had residual LCL laxity. All the ACL avulsions were fixed by the time of ORIF and these were found to be stable at the follow-up. A preoperative MRI could be advisable when treating these fractures, since there is a high prevalence of ligament injuries demanding early operative treatment. Some authors have noted that the severity of associated injuries (vascular, neurological, compartment syndrome) tend to increase as the main fracture line moves laterally [30]. In the current study, we found that most of the LCL ruptures and peroneal nerve injuries were seen in isolated medial condyle fractures, where the lateral condyle was intact. Only six patients (10\%) in the current study developed compartment syndrome.

In one previous study medial plateau fractures were suggested to also carry a high risk for vascular injuries [6]. However, there is not much evidence to support this in the literature. Bennet et al. performed routine arteriography for these patients, but found no vascular injures [31]. In our data from 63 patients with medial plateau fracture, there were no vascular injuries.

The current study had some limitations. Being a cohort study, there were some patients lost to follow-up. Development of posttraumatic OA is multifactorial and in a retrospective study the data is often limited regarding available variables. Since there were different fracture subtypes (split and comminuted), fracture fixation methods were not evaluated, but rather the quality of the reduction. Therefore, we cannot make any recommendation regarding the best fixation method. Only 14 out of 63 patients had preoperative MRI performed, thus we have no comprehensive data on the prevalence of meniscal injuries and only clinical data on the treatment of ligament injuries. Nonetheless, to our knowledge this is the largest study in the English literature examining the outcomes of medial tibial plateau fractures. An important strength of this study was that measurement of articular congruence was performed both preoperatively and at the follow-up visit using CT.

\section{Conclusions}

Good functional outcome can be achieved with ORIF of most medial tibial plateau fractures, but the development of posttraumatic $\mathrm{OA}$ is common. The amount of articular depression as measured from preoperative CT scans seems to predict the development of OA. When treating these fractures, restoration of mechanical axis and articular congruence are important as mechanical axis $>4^{\circ}$ of varus and $\geq 2 \mathrm{~mm}$ articular depression or step-off are associated with worse WOMAC pain scores.

\section{Conflict of interest}

None.

\section{Role of the funding source}

Markus Parkkinen, Tatu Mäkinen and Rami Madanat received research support from the Jane and Aatos Erkko foundation.

\section{Acknowledgements}

The authors would like to thank biostatistician Tero Vahlberg, MSc for performing the statistical analyses.

\section{References}

[1] Schatzker J, McBroom R, Bruce D. The tibial plateau fracture. The Toronto experience 1968-1975. Clin Orthop 1979;94-104.

[2] Purnell ML, Larson AI, Schnetzler KA, Harris NL, Pevny T. Diagnosis and surgical treatment of Schatzker type IV variant biplanar medial tibial plateau fractures in alpine skiers. Tech Knee Surg 2007;6:17-28.

[3] Berkson EM, Virkus WW. High-energy tibial plateau fractures. J Am Acad Orthop Surg 2006;14:20-31.

[4] De Boeck H, Opdecam P. Posteromedial tibial plateau fractures. Clin Orthop Relat Res 1995;330:125-8.

[5] Haider SJ, Pean CA, Davidovitch R, Egol KA. Functional outcomes of isolated medial tibial plateau fractures. J Knee Surg 2016;29:414-22.

[6] Moore TM. Fracture-dislocation of the knee. Clin Orthop Relat Res 1981;12840.

[7] Gardner MJ, Yacoubian S, Geller D, Suk M, Mintz D, Potter H, et al. The insidence of soft tissue injury in operative tibial plateau fractures - a magnetic resonance imaging analysis of 103 patients. J Orthop Trauma 2005;19:79-84.

[8] Honkonen SE. Indications for surgical treatment of tibial condyle fractures. Clin Orthop Relat Res 1994;19:9-205.

[9] Bhattacharyya T, McCarty LP, Harris MB, Morrison SM, Wixted JJ, Vrahas MS, et al. The posterior shearing tibial plateau fracture - treatment and results via posterior approach. J Orthop Trauma 2005;19:305-10.

[10] Koval KJ, Helfet DL. Tibial plateau fractures: evaluation and treatment. J Am Acad Orthop Surg 1995;3:86-94.

[11] Marsh JL, Slongo TF, Agel J, Broderick JS, Creevey W, DeCoster TA, et al. Fracture and dislocation classification compendium - 2007: orthopaedic trauma association classification, database and outcomes committee. J Orthop Trauma 2007;21(Supplement 10):S1-S163.

[12] Pape HC, Rommens PM. AO principles of fracture treatment. 2 ed. Stuttgart ? New York: Thieme; 2007.

[13] Roos EM, Klässbo M, Lohmander LS. WOMAC Osteoarthitis Index. Reliability, validity, and responsiveness in patients with arthroscopically assessed osteoarhritis. Scand J Rheumatol 1999;28:210-5.

[14] Briggs KK, Lysholm J, Tegner Y, Rodkey WG, Kocher MS, Steadman JR. The reliability, validity, and responsiveness of the Lysholm score and Tegner activity scale for anterior cruciate ligament injuries of the knee. Am J Sports Med 2009;37:890-7.

[15] Kellgren JH, Lawrence JS. Radiological assessment of osteo-arthrosis. Ann Rheum Dis 1957;16:494-502.

[16] Rasmussen PS. Tibial condylar fractures as a cause of degenerative arthitis. Acta Orthop Scand 1972;53:566-75.

[17] Honkonen SE. Degenerative arthritis after tibial plateau fractures. J Orthop Trauma 1995;9:273-7.

[18] Rademakers MV, Kerkhoffs GMMJ, Sierevelt IN, Raaymakers ELFB, Marti RK. Operative treatment of 109 tibial plateau fractures - five- to 27-year follow-up results. J Orthop Trauma 2007;21:5-10.

[19] Volpin G, Dowd GS, Stein H, Bentley G. Degenerative arthritis after intraarticular fractures of the knee. J Bone Joint Surg Br 1990;72:634-8.

[20] Wasserstein D, Henry P, Paterson JM, Kreder HJ, Jenkinson R. Risk of total knee arthroplasty after operatively treated tibial plateau fracture: a matchedpopulation-based cohort study. J Bone Joint Surg Am 2014;96:144-50.

[21] Furman BD, Olson SA, Guilak F. The development of posttraumatic arthritis after articular fracture. J Orthop Trauma 2006;20:719-25.

[22] Lotz M. Posttraumatic osteoarthritis: pathogenesis and pharmacological treatment options. Arthritis Res Ther 2010;12:211.

[23] Roos EM. Joint injury causes knee osteoarthritis in young adults. Curr Opin Rheumatol 2005;17:195-200.

[24] Parkkinen M, Madanat R, Mustonen A, Koskinen SK, Paavola M, Lindahl J. Factors predicting the development of early osteoarthritis following lateral tibial plateau fractures: mid-term clinical and radiographic outcomes of 73 operatively treated patients. Scand J Surg 2014;103:256-62.

[25] van der Schoot DKE, Den Outer AJ, Bode PJ, Obermann WR, van Vugt AB. Degenerative changes at the knee and ankle related to malunion of tibial plateau fractures. J Bone Joint Surg Br 1996;78:722-5.

[26] Bellamy N, Wilson C, Hendrikz J. Population-based normative values for the western Ontario and McMaster (WOMAC) osteoarthritis index: part I. Semin Arthritis Rheum 2011;41:139-48.

[27] Parkkinen M, Madanat R, Makinen TJ, Mustonen A, Koskinen SK, Lindahl J. The usefulness of MRI and arthroscopy in the diagnosis and treatment of softtissue injuries associated with split-depression fractures of the lateral tibial condyle. Bone Joint J 2014;96-B:1631-6.

[28] Kannus P. Nonoperative treatment of grade II and III sprains of lateral ligament compartment of the knee. Am J Sports Med 1989;17:83-8.

[29] Geeslin AG, LaPrade RF. Outcomes of treatment of acute grade III isolated and combined posterolateral knee injuries: a prospective case series and surgical techique. J Bone Joint Surg Am 2011;93:1672-83.

[30] Wahlquist M, Iaguilli N, Ebraheim N, Levine J. Medial tibial plateau fractures: a new classification system. J Trauma 2007;63:1418-21.

[31] Bennet WF, Browner B. Tibial plateau fractures - a study of associated soft tissue injuries. J Orthop Trauma 1994;8:183-8. 